\title{
Numerical simulation for SI model with variable-order fractional
}

\author{
Hanaa Abdel Hameed Asfour ${ }^{1}$ and Mohamed Gamal M. Ibrahim ${ }^{2}$ \\ ${ }^{1}$ Doctor of mathematics, Faculty of, Ein Shams University, Egypt \\ ${ }^{2}$ Mathematics Department, Faculty of Science, Zagazig University, Egypt \\ Received: 20 March 2015, Revised: 29 January 2016, Accepted: 12 March 2016 \\ Published online: 10 April 2016
}

\begin{abstract}
In this paper numerical studies for the variable-order fractional delay differential equations are presented. Adams-BashforthMoulton algorithm has been extended to study this problem, where the derivative is defined in the Caputo variable-order fractional sense. Numerical test examples are presented to demonstrate utility of the method. Chaotic behaviors are observed in variable-order one dimensional delayed systems.
\end{abstract}

Keywords: Variable order fractional derivatives, Caputo's derivative, Adams Bashforth-Moulton method, SI model and its Existence and Uniqueness, Solution of SI Model by Using Adams Bashforth-Moulton method.

\section{Introduction}

Variable-order fractional calculus i.e., the fractional differentiation and integration of variable order is the generalization of classical calculus and fractional calculus, which were invented by Newton and Leibnitz hundreds of years ago. Now the study on it becomes a hotpot in recent ten years. It has turned out that many problems in physics, biology, engineering, and finance can be described excellently by models using mathematical tools from variable-order fractional calculus.

In real world systems, delay is very often encountered in many practical systems, such as control systems [1], lasers, traffic models [2], metal cutting, epidemiology, neuro science, population dynamics [3], chemical kinetics [4] etc. Delayed fractional differential equations FDEs are correspondingly used to describe such dynamical systems. In recent years, delayed FDEs begin to arouse the attention of many researchers [5,2]. Simulating these equations is an important technique in the research; accordingly, finding effective numerical methods for the delayed FDEs is a necessary process. The effective methods and their development for numerically solving fractional differential equations (FDEs) have received increasing attention over the last few years. Several methods based on Caputo or Riemann-Liouville definitions [9] have been proposed and analyzed. For instance, based on the predictor-corrector scheme, Diethelm et al. introduced Adams-Bashforth-Moulton algorithm [6,7], and mean while some error analysis presented to improve the numerical accuracy. In recent years, the application of the method is extended to more concrete physical and mathematical models [11]. Variable order differential equations, i.e., differential equations where the order of the derivative changes with respect to either the dependent or the independent variables, have not received as much attention as fractional order systems, despite of the ability of variable order formulations to model continuous spectral behavior in complex dynamics. Many authors have introduced different definitions of variable order differential operators, each of these with a specific meaning to suit desired goals. These definitions such as Riemann-Liouville, Grunwald, Caputo, Riesz ([9],[10]), and some notes as Coimbra definition [8]. The main aim of this paper is to study numerically the variable-order fractional delay differential equations (FDDEs) by using the Adams- Bashforth-Moulton method. 
This paper is organized as follows. In Section 1, we give some definitions and mathematical tools of variable-order fractional calculus. In Section 2, we introduce the known Adams- Bashforth-Moulton method; moreover, the effectiveness of the Adams-Bashforth-Moulton method for solving variable-order fractional differential equation is illustrated. In Section 3, we give an introduction of SI Model and we present the Existence and Uniqueness of SI Model. In section 5 we present solution of SI Model by using Adams-Bashforth-Moulton method, the conclusion is given in Section 6.

\section{Some mathematical tools}

In this part, we give some definitions of fractional derivative and variable-order derivative ([12],[13]).

Definition 1. let $f \in C_{\alpha}$ and $\alpha \geq 0$ then the (left-sided) Riemann-Liouville integral of order $\mu, \mu>0$ is given by

$$
I_{t}^{\mu} f(t)=\frac{1}{\Gamma(\mu)} \int_{0}^{t}(t-\tau) f(\tau) d \tau, t>0
$$

Definition 2. The (left sided) Caputo fractional derivative of $f, f \in C_{-1}^{m}, m \in N \cup\{0\}$, is defined as

$$
D_{t}^{\mu} f(t)=\left\{\begin{array}{l}
\frac{d^{m}}{d t^{m}} f(t), \quad \mu=m \\
I_{t}^{m-\mu} \frac{d^{m} f(t)}{d t^{m}}, m-1 \leq \mu \leq m, m \in N
\end{array}\right.
$$

Note that for $m-1 \leq \mu \leq m, m \in N$,

$$
\begin{gathered}
I_{t}^{\mu} D_{t}^{\mu} f(t)=f(t)-\sum_{k=0}^{m-1} \frac{d^{k} f(0)}{d t^{k}} \frac{t^{k}}{k !} \\
I_{t}^{\mu} t^{v}=\frac{\Gamma(v+1)}{\Gamma(\mu+v+1)} t^{\mu+v} .
\end{gathered}
$$

Definition 3. Let $\alpha(t)$ be a positive real number, $f \in C^{m}[0, T], t \leq$ Tand $m=\left[\max _{0 \leq t \leq T} T\{\alpha(t)\}\right]+1$. Then

$$
D^{\alpha(t)} f(t)=\lim _{h N \rightarrow 0} \frac{1}{h_{N}^{\alpha(t)}} \sum_{k=0}^{N}(-1)^{k}\left(\frac{\alpha(t)}{k}\right) f(t-k h N) .
$$

With $h_{N}=(t-0) / N$ being called the Grunwald-Letnikov variable-order fractional derivative of order $\alpha(t)$ of the function $f$.

Definition 4. The Riemann-Liouville variable order derivative is defined as follows:

$$
D_{t}^{\alpha(t)} f(t)=\frac{1}{\Gamma(m-\alpha(t))}\left(\frac{d^{m}}{d t^{m}}\right) \int_{0}^{t}(t-\tau)^{m-1-\alpha(\tau)} f(\tau) d \tau
$$

Where $\mathrm{m}=\left[\max _{0 \leq t \leq T}\{\alpha(t)\}\right]+1, \mathrm{~m} \in \mathrm{N}$ provided the right side is point wise defined on $\mathrm{t}>0$.

Let $\alpha(\mathrm{t})>0$, be a continuous and bounded function, $\mathrm{f}(\tau) \in \mathrm{C}^{m}[0, \mathrm{t}]$, and $0 \leq \tau \leq \mathrm{t}$. then

$$
\mathrm{D}_{t}^{\alpha(t)} \mathrm{f}(\mathrm{t})= \begin{cases}\frac{1}{\Gamma(m-\alpha(t))} \int_{0}^{t} \frac{f(\tau)}{(t-\tau)^{\alpha(\tau)+1-m}} d \tau, & m-1 \leq \alpha(t) \leq m \\ \frac{d^{m}}{d t^{m}} f(t), & \alpha(t)=m .\end{cases}
$$

Is called the Caputo variable-order fractional derivative of $f(t)$ where $\mathrm{m}=\left[\max _{0 \leq t \leq T}\{\alpha(\mathrm{t})\}\right]+1, \mathrm{~m} \in \mathrm{N}$ and $\Gamma()$ is the Gamma function. 


\section{The Adams-Bash forth-Moulton Method [16]}

In the following we apply the Adams-Bash forth-Moulton predictor-corrector method to implement the numerical solution of variable-order nonlinear FDDEs. Let us consider the following variable-order fractional system:

$$
\begin{gathered}
\mathrm{D}_{t}^{\alpha(t)} \mathrm{y}(\mathrm{t})=\mathrm{f}(\mathrm{y}(\mathrm{t}), \mathrm{y}(\mathrm{t}-\tau)), \mathrm{t} \in[0, \mathrm{~T}], 0<\alpha(\mathrm{t}) \leq 1, \\
\mathrm{y}(\mathrm{t})=\mathrm{g}(\mathrm{t}), \mathrm{t} \in[-\tau, 0]
\end{gathered}
$$

where $f$ is in general a nonlinear function.

Also, consider a uniform grid $\left\{\mathrm{t}_{n}=\mathrm{nh}: \mathrm{n}=-\mathrm{k},-\mathrm{k}+1, . .,-1,0,1, \ldots, \mathrm{N}\right\}$ where $k$ and $N$ are integers such that $h=\tau / k$. Let

$$
\mathrm{y}_{h}\left(t_{j}\right)=\mathrm{g}\left(\mathrm{t}_{j}\right), \mathrm{j}=-\mathrm{k},-\mathrm{k}+1, \ldots,-1,0
$$

And note that

$$
\mathrm{y}_{h}\left(t_{j}-\tau\right)=\mathrm{y}_{h}\left(\mathrm{j}_{\mathrm{h}}-\mathrm{k}_{\mathrm{h}}\right)=\mathrm{y}_{h}\left(\mathrm{t}_{\mathrm{j}-\mathrm{k}}\right), \mathrm{j}=0,1,2, \ldots, \mathrm{N} .
$$

Applying $\mathrm{I}_{t_{\mathrm{n}+1}}^{\alpha\left(t_{\mathrm{n}+1}\right)}$ on both sides of (4) and using (5), we claim to:

$$
\mathrm{y}_{h}\left(\mathrm{t}_{\mathrm{n}+1}\right)=\mathrm{g}(0)+\frac{1}{\Gamma\left(\alpha\left(t_{n+1}\right)\right)} \int_{0}^{t_{n+1}}\left(t_{n+1}-\xi\right)^{\alpha\left(t_{n+1}\right)-1} f(y(\xi), y(\xi-\tau)) d \xi
$$

Further the integral in equation (8) is evaluated using product trapezoidal quadrature formula. Then we have the following corrector formula:

$$
\mathrm{y}_{h}\left(\mathrm{t}_{\mathrm{n}+1}\right)=\mathrm{g}(0)+\frac{h^{\alpha\left(t_{n+1}\right)}}{\Gamma\left(\alpha\left(t_{n+1}\right)+2\right)} f\left(y\left(t_{n+1}\right), y\left(\left(t_{n+1}\right)-\tau\right)\right)+\frac{h^{\alpha\left(t_{n+1}\right)}}{\Gamma\left(\alpha\left(t_{n+1}\right)+2\right)} \sum_{j=0}^{n} a_{j, n+1} f\left(t_{j}, y_{h}\left(t_{j}\right), y_{h}\left(t_{j}-\tau\right)\right)
$$

or

$$
\mathrm{y}_{h}\left(\mathrm{t}_{\mathrm{n}+1}\right)=\mathrm{g}(0)+\frac{h^{\alpha\left(t_{n+1}\right)}}{\Gamma\left(\alpha\left(t_{n+1}\right)+2\right)} f\left(y\left(t_{n+1}\right), y\left(t_{n+1-k}\right)\right)+\frac{h^{\alpha\left(t_{n+1}\right)}}{\Gamma\left(\alpha\left(t_{n+1}\right)+2\right)} \sum_{j=0}^{n} a_{j, n+1} f\left(t_{j}, y_{h}\left(t_{j}\right), y_{h}\left(t_{j-k}\right)\right),
$$

where

$$
\begin{gathered}
a_{j, n+1}=\left\{\begin{array}{l}
n^{\alpha\left(t_{n+1}\right)+1}-\left(n-\alpha\left(t_{n+1}\right)\right)(n+1)^{\alpha\left(t_{n+1}\right)} \\
(n-j+2)^{\alpha\left(t_{n+1}\right)+1}+(n-j)^{\alpha\left(t_{n+1}\right)}-2(n-j+1)^{\alpha\left(t_{n+1}\right)+1} 1 \leq j \geq n, \\
1 \quad j=n+1,
\end{array}\right. \\
y_{h}\left(t_{j-k}\right) \approx v_{n+1}=\left\{\begin{array}{l}
\delta y_{n-m+2}+(1-\delta) y_{n-m+1}, \quad \text { if } m>1 \\
\delta y_{n+1}^{p}+(1-\delta) y_{n}, \quad \text { if } m=1
\end{array}\right.
\end{gathered}
$$

$0 \leq \delta<1$ and the unknown term $\mathrm{y}_{\mathrm{h}}\left(\mathrm{t}_{\mathrm{n}+1}\right)$ appears on both sides of (9). Due to nonlinearity of $f$ equation (9) can't be solved explicitly for $\mathrm{y}_{\mathrm{h}}\left(\mathrm{t}_{\mathrm{n}+1}\right)$, so we replace the term $\mathrm{y}_{\mathrm{h}}\left(\mathrm{t}_{\mathrm{n}+1}\right)$ on the right hand side by an approximation $\mathrm{y}_{\mathrm{h}}^{p}\left(\mathrm{t}_{\mathrm{n}+1}\right)$ which called predictor [15]. The product rectangle rule is used in (8) to evaluate predictor term

$$
\mathrm{y}_{\mathrm{h}}^{p}\left(\mathrm{t}_{\mathrm{n}+1}\right)=g(0)+\frac{1}{\Gamma\left(\alpha\left(t_{n+1}\right)\right)} \sum_{j=0}^{n} b_{j, n+1} f\left(y_{h}\left(t_{j}\right), y_{h}\left(t_{j}-\tau\right)\right)
$$


or

$$
\mathrm{y}_{\mathrm{h}}^{p}\left(\mathrm{t}_{\mathrm{n}+1}\right)=g(0)+\frac{1}{\Gamma\left(\alpha\left(t_{n+1}\right)\right)} \sum_{j=0}^{n} b_{j, n+1} f\left(y_{h}\left(t_{j}\right), y_{h}\left(t_{j-\tau}\right)\right),
$$

where

$$
b_{j, n+1}=\frac{h^{\alpha\left(t_{n+1}\right)}}{\alpha\left(t_{n+1}\right)}\left((n-j+1)^{\alpha\left(t_{n+1}\right)}-(n-j)^{\alpha\left(t_{n+1}\right)}\right) .
$$

Now we apply the mentioned method to determine the numerical solution of variable-order fractional system. Consider the following variable-order fractional system:

$$
\begin{aligned}
& D^{\alpha_{1}(t)} x=f_{1}(x, y, z), \\
& D^{\alpha_{2}(t)} x=f_{2}(x, y, z), \\
& D^{\alpha_{3}(t)} x=f_{3}(x, y, z),
\end{aligned}
$$

With $0<\alpha_{i}(\mathrm{t}) \leq 1$ and initial condition $\left(\mathrm{x}_{0}, \mathrm{y}_{0}, \mathrm{z}_{0}\right)$ According to the previous method, the system (14) can be discredited as follows:

$$
\begin{aligned}
& \mathrm{x}_{\mathrm{n}+1}=x_{0}+\frac{h^{\alpha_{1}\left(t_{n+1}\right)}}{\Gamma\left(\alpha_{1}\left(t_{n+1}\right)+2\right)} f_{1}\left(\mathrm{x}_{n+1}^{p}, y_{n+1}^{p}, z_{n+1}^{p}\right)+\sum \frac{h^{\alpha_{1}\left(t_{n+1}\right)} y_{j, n+1}}{\Gamma\left(\alpha_{1}\left(t_{n+1}\right)+2\right)} f_{1}\left(x_{j}, y_{j}, z_{j}\right), \\
& \mathrm{y}_{\mathrm{n}+1}=y_{0}+\frac{h^{\alpha_{2}\left(t_{n+1}\right)}}{\Gamma\left(\alpha_{2}\left(t_{n+1}\right)+2\right)} f_{2}\left(\mathrm{x}_{n+1}^{p}, y_{n+1}^{p}, z_{n+1}^{p}\right)+\sum \frac{h^{\alpha_{2}\left(t_{n+1}\right)} y_{2 j, n+1}}{\Gamma\left(\alpha_{2}\left(t_{n+1}\right)+2\right)} f_{2}\left(x_{j}, y_{j}, z_{j}\right), \\
& \mathrm{z}_{\mathrm{n}+1}=x_{0}+\frac{h^{\alpha_{3}\left(t_{n+1}\right)}}{\Gamma\left(\alpha_{3}\left(t_{n+1}\right)+2\right)} f_{3}\left(\mathrm{x}_{n+1}^{p}, y_{n+1}^{p}, z_{n+1}^{p}\right)+\sum \frac{h^{\alpha_{3}\left(t_{n+1}\right)} y_{3 j, n+1}}{\Gamma\left(\alpha_{3}\left(t_{n+1}\right)+2\right)} f_{3}\left(x_{j}, y_{j}, z_{j}\right),
\end{aligned}
$$

where

$$
\begin{aligned}
& \mathrm{x}_{n+1}^{p}=x_{0}+\sum \frac{\beta_{1 j, n+1}}{\Gamma\left(\alpha_{1}\left(t_{n+1}\right)\right)} f_{1}\left(x_{j}, y_{j}, z_{j}\right) \\
& \mathrm{y}_{n+1}^{p}=y_{0}+\sum \frac{\beta_{2 j, n+1}}{\Gamma\left(\alpha_{2}\left(t_{n+1}\right)\right)} f_{2}\left(x_{j}, y_{j}, z_{j}\right) \\
& \mathrm{z}_{n+1}^{p}=z_{0}+\sum \frac{\beta_{3 j, n+1}}{\Gamma\left(\alpha_{3}\left(t_{n+1}\right)\right)} f_{3}\left(x_{j}, y_{j}, z_{j}\right) .
\end{aligned}
$$

\section{4- SI model}

The SI Model is the simplest one among the epidemic models. That is why it is also called the Simple Model. We divide the population just in the susceptible compartment $S(t)$ and the infectious compartment $I(t)$. We do assume the disease to be highly infectious but not serious, which means that the ineffective remain in contact with susceptible for all timet $\geq 0$. We also assume that the ineffective continue to spread the disease till the end of the epidemic, the population size to be constant $(S(t)+I(t)=N)$ and homogeneous mixing of population. Infection rate is proportional to the number of infective, i.e. $\beta=r \lambda I$ We have a pair of ordinary differential equations for this model:

$$
\begin{aligned}
& \frac{d S(t)}{d t}=-r \lambda S(t) I(t) \\
& \frac{d I(t)}{d t}=r \lambda S(t) I(t)
\end{aligned}
$$


where

$$
\begin{aligned}
& N=S(t)+I(t) \\
& S(t)=N-I(t)
\end{aligned}
$$

and therefore we get

$$
\frac{d I}{d t}=r \lambda(t)[N-I(t)]
$$

What is known as the logistic growth equation.

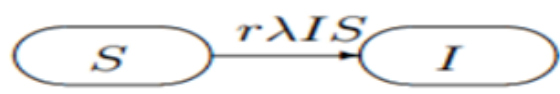

Fig. 1

\subsection{Procedure solution of SI model by using Adams-Bash forth-Moulton method}

At Now, we define the equations (17) at differential order $\alpha(t), \alpha(t)$ then this model can be taking the following form:

$$
\begin{aligned}
& D^{\alpha_{1}(t)} S=-r \lambda S(t) I(t) \\
& D^{\alpha_{2}(t)} S=r \lambda S(t) I(t) .
\end{aligned}
$$

With initial conditions $\mathrm{S}(0)=1, \mathrm{I}(0)=1$ where

$$
\begin{aligned}
& \mathrm{f}_{1}(S, I)=-r \lambda S(t) I(t) \\
& \mathrm{f}_{2}(S, I)=r \lambda S(t) I(t) .
\end{aligned}
$$

By applying the Adams-Bash forth-Moulton Method on SI Model we see the following result

$$
\begin{aligned}
& S_{n+1}=S_{0}+\frac{h^{\alpha_{1}\left(t_{n+1}\right)} y_{j, n+1}}{\Gamma\left(\alpha_{1}\left(t_{n+1}\right)+2\right)} f_{1}\left(S_{n+1}^{p}, I_{n+1}^{p}\right)+\sum \frac{h^{\alpha_{2}\left(t_{n+1}\right)} y_{2 j, n+1}}{\Gamma\left(\alpha_{2}\left(t_{n+1}\right)+2\right)} f_{2}\left(S_{j}, I_{j}\right), \\
& I_{n+1}=I_{0}+\frac{h^{\alpha_{1}\left(t_{n+1}\right)} y_{j, n+1}}{\Gamma\left(\alpha_{1}\left(t_{n+1}\right)+2\right)} f_{1}\left(S_{n+1}^{p}, I_{n+1}^{p}\right)+\sum \frac{h^{\alpha_{2}\left(t_{n+1}\right)} y_{2 j, n+1}}{\Gamma\left(\alpha_{2}\left(t_{n+1}\right)+2\right)} f_{2}\left(S_{j}, I_{j}\right),
\end{aligned}
$$

So

$$
\begin{aligned}
& S_{n+1}=1+\frac{h^{\alpha_{1}\left(t_{n+1}\right)} y_{j, n+1}}{\Gamma\left(\alpha_{1}\left(t_{n+1}\right)+2\right)}\left(-r \lambda S_{n+1}^{p} I_{n+1}^{p}\right)+\sum \frac{h^{\alpha_{2}\left(t_{n+1}\right)} y_{2 j, n+1}}{\Gamma\left(\alpha_{2}\left(t_{n+1}\right)+2\right)}\left(-r \lambda S_{j} I_{j}\right) \\
& I_{n+1}=1+\frac{h^{\alpha_{1}\left(t_{n+1}\right)} y_{j, n+1}}{\Gamma\left(\alpha_{1}\left(t_{n+1}\right)+2\right)}\left(-r \lambda S_{n+1}^{p} I_{n+1}^{p}\right)+\sum \frac{h^{\alpha_{2}\left(t_{n+1}\right)} y_{2 j, n+1}}{\Gamma\left(\alpha_{2}\left(t_{n+1}\right)+2\right)}\left(r \lambda S_{j} I_{j}\right) .
\end{aligned}
$$

Figs. 1 and 2 show the solutions $S(t)$ and $I(t)$ of system (17) for $\alpha=1, \mathrm{~h}=0.1$ and $\alpha=0.5, \mathrm{~h}=0.1$. 


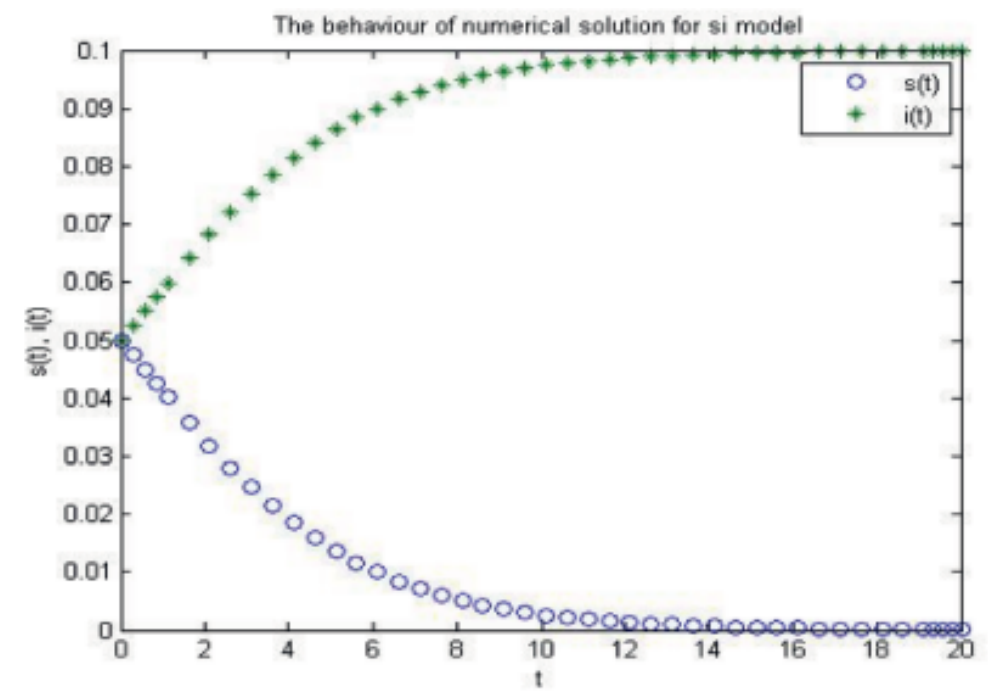

Fig. 2: The numerical behavior os system (17) at $\alpha=1$ and $h=0.1$.

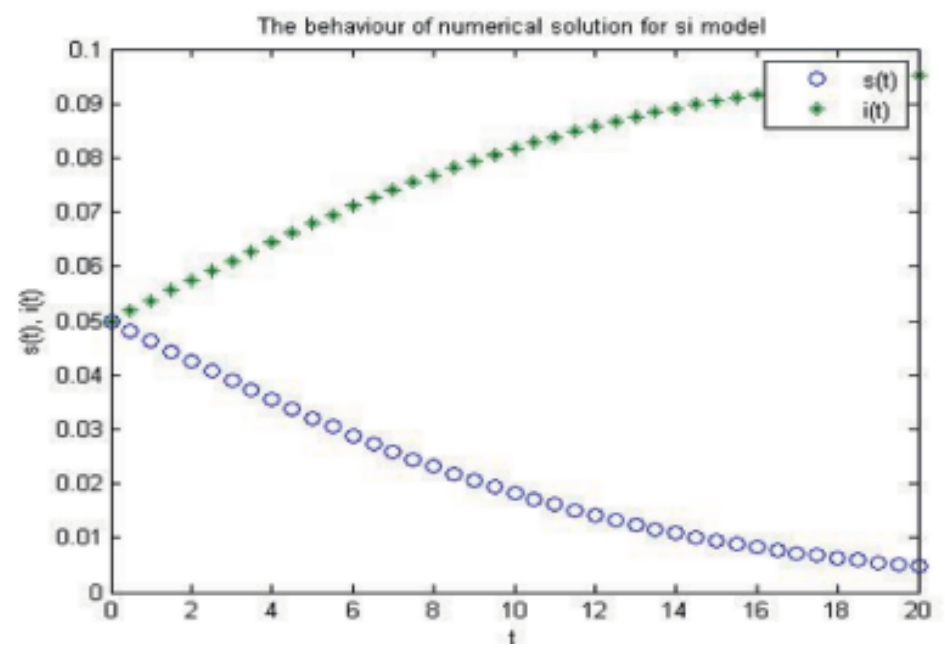

Fig. 3: The numerical behavior os system (17) at $\alpha=0.5$ and $h=0.1$.

\subsection{Convergent of SI model}

Using equation (17), put $\alpha=\alpha=\alpha$ and use

$$
D^{\alpha_{1}} S(t)=\sigma_{\alpha, k} \sum_{j=0}^{n} \omega_{j}^{(\alpha)}\left[S_{n-j+1}-S_{n-j}\right]
$$

and

$$
D^{\alpha_{2}} S(t)=\sigma_{\alpha, k} \sum_{j=0}^{n} \omega_{j}^{(\alpha)}\left[I_{n-j+1}-I_{n-j}\right]
$$


in [14]. Then by using the latest equations. So we have got

$$
\begin{array}{ll}
\sigma_{\alpha, k} \sum_{j=0}^{n} \omega_{j}^{(\alpha)}\left[S_{n-j+1}-S_{n-j}\right]+r \lambda S_{n} I_{n}=0 & F_{1} \\
\sigma_{\alpha, k} \sum_{j=0}^{n} \omega_{j}^{(\alpha)}\left[I_{n-j+1}-I_{n-j}\right]-r \lambda S_{n} I_{n}=0 & F_{2} .
\end{array}
$$

We substituting for value of $n=1,2,3, \ldots$, at $n=1$ equation (24) become

$$
\begin{aligned}
& \sigma_{\alpha, k}\left(\omega_{1}^{(\alpha)}\left[S_{1}-S_{0}\right]\right)+r \lambda S_{1} I_{1}=0 \\
& \sigma_{\alpha, k}\left(\omega_{1}^{(\alpha)}\left[I_{1}-I_{0}\right]\right)-r \lambda S_{1} I_{1}=0
\end{aligned}
$$

By using Jacobin matrix

$$
\left(J_{n}\right)=\left(\begin{array}{ll}
\frac{\partial F_{1}}{\partial S_{n}} & \frac{\partial F_{2}}{\partial S_{n}} \\
\frac{\partial F_{1}}{\partial I_{n}} & \frac{\partial F_{1}}{\partial I_{n}}
\end{array}\right)
$$

Use (25) and (26) to evaluate

$$
\left|J_{1}\right|=\left(\begin{array}{ll}
\frac{\partial F_{1}}{\partial S_{1}} & \frac{\partial F_{2}}{\partial S_{1}} \\
\frac{\partial F_{1}}{\partial I_{1}} & \frac{\partial F_{2}}{\partial I_{1}}
\end{array}\right)=\left(\begin{array}{cc}
\sigma_{\alpha, k} \omega_{1}^{(\alpha)}+r \lambda I_{1} & -r \lambda I_{1} \\
r \lambda S_{1} & \sigma_{\alpha, k} \omega_{1}^{(\alpha)}+r \lambda S_{1}
\end{array}\right)
$$

So the determinant of Jacobin matrix equal to

$$
\begin{aligned}
&\left|J_{1}\right|=\left|\begin{array}{cc}
\sigma_{\alpha, k} \omega_{1}^{(\alpha)}+r \lambda I_{1} & -r \lambda I_{1} \\
r \lambda S_{1} & \sigma_{\alpha, k} \omega_{1}^{(\alpha)}+r \lambda S_{1}
\end{array}\right|=\left(\sigma_{\alpha, k} \omega_{1}^{(\alpha)}+r \lambda I_{1}\right)\left(\sigma_{\alpha, k} \omega_{1}^{(\alpha)}-r \lambda S_{1}\right)+r^{2} \lambda^{2} S_{1} I_{1} \\
& \quad=\sigma_{\alpha, k}^{2} \omega_{1}^{2(\alpha)}+\sigma_{\alpha, k} \omega_{1}^{(\alpha)}\left(r \lambda I_{1}-r \lambda S_{1}\right)-r^{2} \lambda^{2} S_{1} I_{1}+r^{2} \lambda^{2} S_{1} I_{1}=\sigma_{\alpha, k}^{2} \omega_{1}^{2(\alpha)}+\sigma_{\alpha, k} \omega_{1}^{(\alpha)}\left(r \lambda I_{1}-r \lambda S_{1}\right)(i) .
\end{aligned}
$$

At $n=2$ equation (24) become

$$
\begin{aligned}
& \sigma_{\alpha, k}\left(\omega_{1}^{(\alpha)}\left[S_{1}-S_{0}\right]+\omega_{2}^{(\alpha)}\left[S_{2}-S_{1}\right]\right)+r \lambda S_{2} I_{2}=0 \\
& \sigma_{\alpha, k}\left(\omega_{1}^{(\alpha)}\left[I_{1}-I_{0}\right]+\omega_{2}^{(\alpha)}\left[I_{2}-I_{1}\right]\right)-r \lambda S_{2} I_{2}=0
\end{aligned}
$$

By using Jacobin matrix

$$
\left(J_{2}\right)=\left(\begin{array}{ll}
\frac{\partial F_{1}}{\partial S_{2}} & \frac{\partial F_{2}}{\partial S_{2}} \\
\frac{\partial F_{1}}{\partial I_{2}} & \frac{\partial F_{1}}{\partial I_{2}}
\end{array}\right)=\left(\begin{array}{cc}
\sigma_{\alpha, k} \omega_{2}^{(\alpha)}+r \lambda I_{2} & -r \lambda I_{2} \\
r \lambda S_{2} & \sigma_{\alpha, k} \omega_{2}^{(\alpha)}+r \lambda S_{2}
\end{array}\right)
$$

So the determinant of Jacobin matrix equal to

$$
\begin{array}{r}
\left|J_{2}\right|=\left|\begin{array}{cc}
\sigma_{\alpha, k} \omega_{2}^{(\alpha)}+r \lambda I_{2} & -r \lambda I_{2} \\
r \lambda S_{2} & \sigma_{\alpha, k} \omega_{2}^{(\alpha)}+r \lambda I_{2}
\end{array}\right|=\left(\sigma_{\alpha, k} \omega_{2}^{(\alpha)}+r \lambda I_{2}\right)\left(\sigma_{\alpha, k} \omega_{2}^{(\alpha)}-r \lambda S_{2}\right)+r^{2} \lambda^{2} S_{2} I_{2} \\
=\sigma_{\alpha, k}^{2} \omega_{2}^{2(\alpha)}+\sigma_{\alpha, k} \omega_{2}^{(\alpha)}\left(r \lambda I_{2}-r \lambda S_{2}\right)-r^{2} \lambda^{2} S_{2} I_{2}+r^{2} \lambda^{2} S_{2} I_{2}=\sigma_{\alpha, k}^{2} \omega_{2}^{2(\alpha)}+\sigma_{\alpha, k} \omega_{2}^{(\alpha)}\left(r \lambda I_{2}-r \lambda S_{2}\right)(i i),
\end{array}
$$

and so on 
At $n$ equation (24) become

$$
\begin{aligned}
& \sigma_{\alpha, k}\left(\omega_{1}^{(\alpha)}\left[S_{1}-S_{0}\right]+\ldots+\omega_{n}^{(\alpha)}\left[S_{n}-S_{n-1}\right]\right)+r \lambda S_{n} I_{n}=0 \\
& \sigma_{\alpha, k}\left(\omega_{1}^{(\alpha)}\left[I_{1}-I_{0}\right]+\ldots+\omega_{n}^{(\alpha)}\left[I_{n}-I_{n-1}\right]\right)+r \lambda S_{n} I_{n}=0
\end{aligned}
$$

By using Jacobin matrix

$$
\left(J_{n}\right)=\left(\begin{array}{ll}
\frac{\partial F_{1}}{\partial S_{n}} & \frac{\partial F_{2}}{\partial S_{n}} \\
\frac{\partial F_{1}}{\partial I_{n}} & \frac{\partial F_{1}}{\partial I_{n}}
\end{array}\right)=\left(\begin{array}{cc}
\sigma_{\alpha, k} \omega_{n}^{(\alpha)}+r \lambda I_{n} & -r \lambda I_{n} \\
r \lambda S_{n} & \sigma_{\alpha, k} \omega_{n}^{(\alpha)}+r \lambda S_{n}
\end{array}\right)
$$

So the determinant of Jacobin matrix equal to

$$
\begin{aligned}
\left|J_{n}\right| & =\left|\begin{array}{cc}
\sigma_{\alpha, k} \omega_{n}^{(\alpha)}+r \lambda I_{n} & -r \lambda I_{n} \\
r \lambda S_{n} & \sigma_{\alpha, k} \omega_{n}^{(\alpha)}+r \lambda I_{n}
\end{array}\right|=\left(\sigma_{\alpha, k} \omega_{n}^{(\alpha)}+r \lambda I_{n}\right)\left(\sigma_{\alpha, k} \omega_{n}^{(\alpha)}-r \lambda S_{n}\right)+r^{2} \lambda^{2} S_{n} I_{n} \\
= & \sigma_{\alpha, k}^{2} \omega_{n}^{2(\alpha)}+\sigma_{\alpha, k} \omega_{n}^{(\alpha)}\left(r \lambda I_{n}-r \lambda S_{n}\right)-r^{2} \lambda^{2} S_{n} I_{n}+r^{2} \lambda^{2} S_{n} I_{n}=\sigma_{\alpha, k}^{2} \omega_{2}^{2(\alpha)}+\sigma_{\alpha, k} \omega_{2}^{(\alpha)}\left(r \lambda I_{2}-r \lambda S_{2}\right)(i i i)
\end{aligned}
$$

By $((i),(i i),(i i i)) S I$ model convergent uniformly If $\left|J_{n}\right|=0$ then weather or $\sigma_{\alpha, k} \omega_{n}^{(\alpha)}=0$.

$$
\begin{aligned}
& \sigma_{\alpha, k} \omega_{n}^{(\alpha)}\left(r \lambda I_{n}-r \lambda S_{n}\right)=0 \\
& \sigma_{\alpha, k} \omega_{n}^{(\alpha)}=r \lambda\left(I_{n}-S_{n}\right)
\end{aligned}
$$

In this section, we study the numerical solution of the non-linear fractional SI model of the form:

$$
\begin{aligned}
& D^{\alpha_{1}(t)} S=-r \lambda S(t) I(t), \quad t>0,0<\alpha_{1}, \alpha_{2} \leq 1 \\
& D^{\alpha_{2}(t)} S=r \lambda S(t) I(t)
\end{aligned}
$$

The parameter $\alpha(t), \alpha(t)$ refers to the fractional order of the time derivative. With initial condition.

$$
S(0)=S_{0}, I(0)=I_{0}
$$

\subsection{Existence and uniqueness of SI model}

Let $\mathrm{J}=[0,1], \mathrm{T}<\infty$ and $\mathrm{C}(\mathrm{J})$ be the class of all continuous functions defined on $J$, with the norm. $\|S\|=\sup _{t \in J}\left|e^{-N t} S(t)\right|$, $\|I\|=\sup _{t \in J}\left|e^{-N t} I(t)\right|, N>0$, which is equivalent to the sup-norm $\|S\|=\sup _{t \in J}|s(t)|,\|I\|=\sup _{t \in J}|I(t)|$. To study the existence and the uniqueness of the initial value problem of the fractional SI model (30), we suppose that the solution $\mathrm{S}(\mathrm{t}), \mathrm{I}(\mathrm{t})$ belongs to the space $B=\{S, I \in \mathfrak{R}:|S, I| \leq b$ for any constant $b$ The initial Value problem (32) has a unique solution $S, I \in C(J)$.

$$
S^{l}, I^{l} \in X=\left\{S, I \in L[0,1],\|S\|=\left\|e^{-N t} S(t)\right\| L_{1},\|I\|=\left\|e^{-N t} S(t)\right\| L_{1}\right\}
$$

Proof. Form properties of fractional calculus. The fractional differential equation (30) can be written as.

$$
\begin{aligned}
& \mathrm{I}^{1-\alpha} \frac{d S(t)}{d t}=-r \lambda S(t) I(t) \\
& \mathrm{I}^{1-\alpha} \frac{d I(t)}{d t}=r \lambda S(t) I(t) .
\end{aligned}
$$


Operating with $\mathrm{I}^{\alpha}$ so we obtain

$$
\begin{aligned}
& S(t)=\mathrm{I}^{\alpha}[-r \lambda S(t) I(t)] \\
& I(t)=\mathrm{I}^{\alpha}[r \lambda S(t) I(t)] .
\end{aligned}
$$

Now let us defined the operator $\mathrm{F}: \mathrm{C}(\mathrm{J}) \rightarrow \mathrm{C}(\mathrm{J})$ by

$$
\begin{aligned}
& F S(t)=\mathrm{I}^{\alpha}[-r \lambda S(t) I(t)] \\
& F I(t)=\mathrm{I}^{\alpha}[r \lambda S(t) I(t)] .
\end{aligned}
$$

Then

(1)

$$
\begin{aligned}
& e^{-N t}\left(F S_{1}-F S_{2}\right)=e^{-N t} \mathrm{I}^{\alpha}\left[-r \lambda S_{1}(t) I(t)+r \lambda S_{2}(t) I(t)\right] \\
& =e^{-N t} \mathrm{I}^{\alpha} r \lambda I(t)\left[S_{2}(t)-S_{1}(t)\right] \\
& \leq \int_{0}^{t} \frac{(t-S)^{\alpha-1}}{\Gamma(\alpha)} e^{N(t-s)}(-r \lambda) \mathrm{I}^{\alpha} I(t)\left[S_{2}(t)-S_{1}(t)\right] e^{-N s} d s \\
& \leq\left\|I_{2}-I_{1}\right\| \int_{0}^{t} \frac{-r \lambda S^{\alpha-1} e^{-N s}}{\Gamma(\alpha)} d s
\end{aligned}
$$

There for we obtain

$$
e^{-N t}\left(F S_{1}-F S_{2}\right) \leq\left\|S_{2}-S_{1}\right\|
$$

(2)

$$
\begin{aligned}
e^{-N t}\left(F I_{1}\right. & \left.-F I_{2}\right)=e^{-N t} \mathrm{I}^{\alpha}\left[-r \lambda I_{1}(t) S(t)+r \lambda I_{2}(t) S(t)\right] \\
& =e^{-N t} \mathrm{I}^{\alpha} r \lambda S(t)\left[I_{2}(t)-I_{1}(t)\right] \\
& \leq \int_{0}^{t} \frac{(t-S)^{\alpha-1}}{\Gamma(\alpha)} e^{N(t-s)}(-r \lambda) \mathrm{I}^{\alpha} S(t)\left[I_{2}(t)-I_{1}(t)\right] e^{-N s} d s \\
& \leq\left\|I_{2}-I_{1}\right\| \int_{0}^{t} \frac{-r \lambda S^{\alpha-1} e^{-N s}}{\Gamma(\alpha)} d s .
\end{aligned}
$$

There for we obtain

$$
e^{-N t}\left(F I_{1}-F I_{2}\right) \leq\left\|I_{2}-I_{1}\right\| .
$$

And operator F given by (35) has a unique fixed point. Consequently the integral equation (32) has a unique solution, $S, I \in C(J)$.

Now from equation (32), we formally have

(1)

$$
S(t)=\frac{t^{\alpha}}{\Gamma(\alpha+1)}\left[\left(-r \lambda S_{0} I\right)+\mathrm{I}^{\alpha}\left(-r \lambda S^{l} I\right)\right],
$$

and

$$
\begin{aligned}
& \frac{d S(t)}{d t}=\frac{t^{\alpha-1}}{\Gamma(\alpha+1)}\left[\left(-r \lambda S_{0} I\right)+\mathrm{I}^{\alpha}\left(-r \lambda S^{l} I\right)\right] \\
& e^{-N t} S^{l}(t)=e^{-N t}\left[\frac{t^{\alpha-1}}{\Gamma(\alpha+1)}\left[\left(-r \lambda S_{0} I\right)+\mathrm{I}^{\alpha}\left(-r \lambda S^{l} I\right)\right]\right] .
\end{aligned}
$$


From which we can deduce that $S^{l} \in \mathrm{C}(\mathrm{J})$ and $S^{l} \in \mathrm{X}$ Now from Eq.(35), we get

$$
\begin{aligned}
& \frac{d S(t)}{d t}=\frac{d}{d t} \mathrm{I}^{\alpha}[-r \lambda S I] \\
& \mathrm{I}^{\alpha-1} \frac{d S(t)}{d t}=\mathrm{I}^{1-\alpha} \frac{d}{d t}[-r \lambda S I]=\frac{d}{d t} \mathrm{I}^{1-\alpha} \mathrm{I}^{\alpha}[-r \lambda S I] \\
& D^{\alpha} S(t)=\frac{d}{d t} \mathrm{I}[-r \lambda S I]=-r \lambda S I,
\end{aligned}
$$

and

$$
S(0)=\mathrm{I}^{\alpha}[-r \lambda S I]_{t=0}=0
$$

(2)

$$
I(t)=\frac{t^{\alpha}}{\Gamma(\alpha+1)}\left[\left(-r \lambda S I_{0}\right)+I^{\alpha}\left(-r \lambda S I^{l}\right)\right]
$$

and

$$
\begin{aligned}
& \frac{d I(t)}{d t}=\frac{t^{\alpha-1}}{\Gamma(\alpha+1)}\left[\left(-r \lambda S I_{0}\right)+\mathrm{I}^{\alpha}\left(-r \lambda S I^{l}\right)\right] \\
& e^{-N t} I^{l}(t)=e^{-N t}\left[\frac{t^{\alpha-1}}{\Gamma(\alpha+1)}\left[\left(-r \lambda S I^{l}\right)+\mathrm{I}^{\alpha}\left(-r \lambda S I^{l}\right)\right]\right] .
\end{aligned}
$$

From which we can deduce that $\mathrm{I}^{l} \in \mathrm{C}(\mathrm{J})$ and $\mathrm{I}^{l} \in \mathrm{X}$ now from Eq. (35), we get

$$
\begin{aligned}
& \frac{d I(t)}{d t}=\frac{d}{d t} \mathrm{I}^{\alpha}[r \lambda S I], \\
& \mathrm{I}^{\alpha-1} \frac{d I(t)}{d t}=\mathrm{I}^{1-\alpha} \frac{d}{d t}[r \lambda S I]=\frac{d}{d t} \mathrm{I}^{1-\alpha} \mathrm{I}^{\alpha}[r \lambda S I], \\
& D^{\alpha} I(t)=\frac{d}{d t} \mathrm{I}[r \lambda S I]=r \lambda S I,
\end{aligned}
$$

and

$$
I(0)=\mathrm{I}^{\alpha}[r \lambda S I]_{t=0}=0 .
$$

Then the integral equation (32) is equivalent to the initial value problem (31) and theorem is proved.

\section{Conclusions}

Variable-order fractional calculus has been highly neglected since it was proposed. Nevertheless, the scientific community has found a large variety of applications which can be modeled and more clearly understood by using this branch of mathematics. By using the Adams-Bash forth-Moulton method, we obtain the numerical solution of the variable-order fractional SI Model We present a convergent of SI Model in section 4, also we prove that the Existence and Uniqueness of SI Model. Moreover, we hope our work about variable-order fractional calculus would generate interest from related scholars in the future and also hope that their work may result in significant contributions to this field.

\section{References}

[1] E. Fridman, L. Fridman and E. Shustin, Steady modes in relay control systems with time delay and periodic disturbances, J. Dyn. Sys., Meas., Control, 122(4), 732-737, 2000. 
[2] L. C. Davis, Modification of the optimal velocity traffic model to include delay due to driver reaction time, Physica A, 319 , 557-567, 2002.

[3] Y. Kuang, Delay differential equations with applications in population biology, Academic Press, Boston, San Diego, New York, 1993.

[4] I. Epstein and Y. Luo, Differential delay equations in chemical kinetics. Nonlinear models: the cross-shaped phase diagram and the oregonator, J. Chem. Phys., 95, 244-254, 1991.

[5] S. Bhalekar, V. Daftardar-Gejji, A predictor-corrector scheme for solving nonlinear delay differential equations of fractional order, Journal of Fractional Calculus and Applications, 1(5), 1-9, 2011.

[6] K. Diethelm, The Analysis of Fractional Differential Equations, Springer, Berlin, Germany, 2010.

[7] K. Diethelm, N. J. Ford, and A. D. Freed, A predictor-corrector approach for the numerical solution of fractional differential equations, Nonlinear Dynamics, 29, 3-22, 2002.

[8] C. F. M. Coimbra, Mechanics with variable-order differential operators, Annulet der Physic, 12(11-12), 692-703, 2003.

[9] C. F. Lorenzo, T.T. Hartley, The vector linear fractional initialization problem, National Aeronautics and Space Administration, Glenn Research Center, 1999.

[10] R. Lin, F. Liu , V. Anh, I. Turner, Stability and convergence of a new explicit FDM for the variable-order nonlinear fractional diffusion equation, Applied Mathematics and Computation, 212, 435-445, 2009.

[11] S. Ma, Y. Xu, and W. Yue, Numerical solutions of a variable-order fractional financial system, Hindawi Publishing Corporation Journal of Applied Mathematics, 14, 1-15, 2012.

[12] P. L. Butzer and U.Westphal, An introduction to fractional calculus, World Scientific, Singapore, 2000.

[13] S. Umarov and S. Steinberg, Variable order differential equations with piecewise constant order function and diffusion with changing modes, Zeitschrift Analysis and Anwendungen, 28(4), 131-150, 2009.

[14] N. H. Sweilam, A. M. Nagy, T. A. Assiri and N.Y.Ali, Numerical Simulations For Variable-Order Fractional Nonlinear Delay Differential equations Journal of Fractional Calculus and Applications,6(1) Jan. 2015, pp. 71-82.

[15] M. M. Khader, N. H. Sweilam, A. M. S. Mahdy and N. K. Abdel Moniem, Numerical Simulation for the Fractional SIRC Model and Influenza A, Applied Mathematics \& Information Sciences Appl. Math. Inf. Sci. 3, 1-8 (2014).

[16] N. H. Sweilam, A. M. Nagy, T. A. Assiri and N.Y.Ali, Numerical simulations for Variable-order fractional nonlinear delay differential equations, 6, 71-82, 2015. 